\title{
The Bivariate Pareto Model Based on Ordered Random Variables
}

\author{
Devendra Kumar
}

(Communicated by Türkan ERBAY DALKILIÇ)

\begin{abstract}
Generalized order statistics constitute a unified model for ordered random variables that includes order statistics and record values among others. In this article, bivariate Pareto distribution is considered. Some new simple explicit expressions for single and product moments of concomitants of generalized order statistics based on a random sample drown from the considered distribution are derived. Further, applications of these results is seen in establishing some well known results given separately for order statistics and record values and obtaining some new results. Finally, the means, and variances of the concomitants of order statistics and record values are computed for various values of the parameters.
\end{abstract}

Keywords: Generalized order statistics; order statistics; upper record values; bivariate Pareto distribution; concomitants.

AMS Subject Classification (2010): Primary: 62G30; 62E10.

\section{Introduction}

Pareto distributions have been extensively employed for modeling and analysis of statistical data under different contexts. Orginally, the distribution was first proposed as a model to explain the allocation of wealth among individuals. Later, various forms of the Pareto distribution have been formulated for modeling and analysis of data from engineering, environment, geology, hydrology etc. These diverse applications of the Pareto distributions lead researchers to develop different kinds of bivariate (multivariate) Pareto distributions. Accordingly, Mardia (1962) introduced two types of bivariate (multivariate) Pareto models which are referred as bivariate Pareto distributions of first kind and second kind respectively. Since then there has been a lot of works in the form, alternative derivation of bivariate Pareto models, their extensions, inference, characterizations and applications to a variety of fields. The literature on bivariate (multivariate) Pareto model is vast, disjoint, and scattered across many different journals. See for example Lindley and Singpurwalla (1986), Arnold (1985), Arnold (1990), Sankaran and Nair (1993), Hutchinson and Lai (1990), Langseth (2002), Balakrishnan and Lai (2009) and Sankaran and Kundu (2014), Sankaran et al (2015), among others have extended the use of the bivariate Pareto models to other branches of statistics, such as reliability, risk and quality control.

Lindley and Singpurwalla (1986) proposed the bivariate Pareto distribution, which was further studied by Nayak (1987), Sankaran and Nair (1993), Langseth (2002), Balakrishnan and Lai (2009), Sankaran and Kundu (2014) and Sankaran et al. (2015). Nayak (1987) Proposed multivariate lomax distribution, discuss several properties and usefulness in reliability theory. Sankaran and Nair (1993) proposed a bivariate Pareto distribution and discuss the different applications in reliability set up, and finally propose a multivariate generalization. Langseth (2002) investigate the mathematical modelling of maintenance and repair of components that can fail due to a variety of failure mechanisms. Balakrishnan and Lai (2009) studied the Continuous bivariate distributions and describes in detail their forms, properties, dependence structures, computation, and applications. Sankaran and Kundu (2014)

Received : 07-June-2016, Accepted : 21-October-2016 
obtained maximum likelihood estimates of the parameters of bivariate Pareto model. Further, They observed that this model can used quite effectively to analyze competing risk data. Sankaran et al. (2015) have characterized a family of bivariate Pareto distributions through a generalized version of the univariate dullness property.

Order statistics and functions of these statistics play an important role in a wide range of theoretical and practical problems such as characterization of probability distributions and goodness-of-fit tests, entropy estimation, analysis of censored samples, reliability analysis, quality control and strength of materials; see Arnold et al. (1992) and David and Nagaraja (2003) and the references therein for more details. The practicability of moments of order statistics can be seen in many areas such as quality control testing, reliability, etc. For instance, when the reliability of an item or product is high, the duration of the failed items will be high which in turn will make the product too expensive, both in terms of time and money. This fact prevents a practitioner from knowing enough about the product in a relatively short time. Therefore, a practitioner needs to predict the failure of future items based on the times of a few early failures. These predictions are often based on moments of order statistics.

Recently, Balakrishnan et al. (2015) established some recurrence relations for single and product moments of order statistics of the complementary exponential-geometric distribution. The computation of moments of order statistics is a challenging task for many distributions. For this reason, recursive computational methods are often sought.

A random variable $X$ has the bivariate Pareto distribution with parameters $\alpha_{1}, \alpha_{2}$ and $\theta$, if its probability density function $(p d f)$ is

$$
f(x, y)=\theta(\theta+1) \alpha_{1} \alpha_{2}\left(1+\alpha_{1} x+\alpha_{2} y\right)^{-(\theta+2)}, x, y>0, \alpha_{1}, \alpha_{2}, \theta>0
$$

the corresponding cumulative distribution function $(c d f)$ is

$$
F(x, y)=1-\left(1+\alpha_{1} x+\alpha_{2} y\right)^{-\theta}, x, y>0, \alpha_{1}, \alpha_{2}, \theta>0 .
$$

The conditional $p d f$ of $Y$ given $X$ is

$$
f(y \mid x)=\frac{\alpha_{2}(\theta+1)\left(1+\alpha_{1} x\right)^{\theta+1}}{\left(1+\alpha_{1} x+\alpha_{2} y\right)^{\theta+2}}, y>0,
$$

the corresponding marginal $p d f$ and $c d f$ are

$$
f(x)=\theta \alpha_{1}\left(1+\alpha_{1} x\right)^{-(\theta+1)}, x>0,
$$

and

$$
F(x)=1-\left(1+\alpha_{1} x\right)^{-\theta}, x>0 .
$$

Various developments on generalized order statistics (GOS) and related topics have been studied by Kamps and Gather (1997), Ahsanullah (2000), Pawlas and Szynal (2001), Kamps and Cramer (2001), Ahmad and Fawzy (2003), Ahmad (2007), Kumar (2010, 2011 and 2013) among others. Kumar (2015a, 2015b ) have established the exact moments of GOS from type II exponentiated log-logistic distribution and lower generalized order statistics based on inverse Burr distribution respectively. Characterizations based on GOS have been studied by some authors, Keseling (1999) characterized some continuous distributions based on conditional distributions of GOS. Bieniek and Szynal (2003) characterized some distributions via linearity of regression of GOS. Cramer et al. (2004) gave a unifying approach on characterization via linear regression of ordered random variables.

Rest of the paper is organized as follows: In Section 2, we describe briefly the preliminaries of GOS. In Section 3, we present the marginal $p d f$ of concomitant of GOS from bivariate Pareto distribution. In Section 4, we obtained some new explicit expressions for single moments of concomitants of GOS from bivariate Pareto distribution. In Section 5, we discussed the joint of concomitant of GOS from bivariate Pareto distribution. In Section 6, we obtained the explicit expressions for product moments of concomitant of GOS from bivariate Pareto distribution. Tabulations of means, variances and covariances of order statistics are given in Section 7. Two applications are performed in Section 8. Finally, in Section 9, we make some concluding remarks.

\section{Generalized order statistics and preliminaries}

The concept of generalized order statistics (GOS) was introduced by Kamps (1995). Several models of ordered random variables such as order statistics, record values, sequential order statistics, progressive type II censored order statistics and Pfeifer's record values can be discussed as special cases of the GOS. Suppose $X(1, n, m, k), \ldots$, 
$X(n, n, m, k),(k$ is a positive integer and $m$ is a real number), are $n$ GOS from an absolutely continuous cumulative distribution function $F(x)$ with probability density function $f(x)$, if their joint $p d f$ is of the form

$$
k\left(\prod_{j=1}^{n-1} \gamma_{j}\right)\left(\prod_{i=1}^{n-1}\left[1-F\left(x_{i}\right)\right]^{m} f\left(x_{i}\right)\right)\left[1-F\left(x_{n}\right)\right]^{k-1} f\left(x_{n}\right)
$$

on the cone $F^{-1}(0) \leq x_{1} \leq x_{2} \leq \ldots \leq x_{n} \leq F^{-1}(1)$, where $\gamma_{j}=k+(n-j)(m+1)>0$ for all $j, 1 \leq j \leq n, k$ is a positive integer and $m \geq-1$.

If $m=0$ and $k=1$, then this model reduces to the ordinary $r^{t h}$ order statistic and (2.1) will be the joint $p d f$ of $n$ order statistics $X_{1: n} \leq X_{2: n} \leq \ldots \leq X_{n: n}$ from $c d f F(x)$. If $m=-1$ and $k=1$, then (2.1) will be the joint $p d f$ of the first $n$ record values of the identically and independently distributed (iid) random variables with $c d f F(x)$ and corresponding $p d f f(x)$.

In view of (2.1), the marginal $p d f$ of the $r^{t h} \mathrm{GOS}, X(r, n, m, k), 1 \leq r \leq n$, is

$$
f_{X(r, n, m, k)}(x)=\frac{C_{r-1}}{(r-1) !}[\bar{F}(x)]^{\gamma_{r}-1} f(x) g_{m}^{r-1}(F(x))
$$

and the joint $p d f$ of $X(r, n, m, k)$ and $X(s, n, m, k), 1 \leq r<s \leq n$, is

$$
\begin{gathered}
f_{X(r, n, m, k), X(s, n, m, k)}(x, y)=\frac{C_{s-1}}{(r-1) !(s-r-1) !}[\bar{F}(x)]^{m} f(x) g_{m}^{r-1}(F(x)) \\
\times\left[h_{m}(F(y))-h_{m}(F(x))\right]^{s-r-1}[\bar{F}(y)]^{\gamma_{s}-1} f(y), \quad x<y,
\end{gathered}
$$

where

$$
\begin{gathered}
\bar{F}(x)=1-F(x), \quad C_{r-1}=\prod_{i=1}^{r} \gamma_{i}, \quad \gamma_{i}=k+(n-i)(m+1), \\
h_{m}(x)= \begin{cases}-\frac{1}{m+1}(1-x)^{m+1}, & m \neq-1 \\
-\ln (1-x), & m=-1\end{cases}
\end{gathered}
$$

and

$$
g_{m}(x)=h_{m}(x)-h_{m}(1), \quad x \in[0,1) .
$$

Let a random sample $\left(X_{i}, Y_{i}\right), i=1,2, \ldots$ be a sequence of $i . i . d$. from a bivariate random variables $(X, Y)$ with an absolutely continuous $c d f F(x, y)$. If the pairs are ordered by their $X$ variates, then the $Y$ variates associated with $r^{t h} \operatorname{GOS} X(r, n, m, k)$ of $X$ will be denoted by $Y_{[r, n, m, k]}, 1 \leq r \leq n$ and called the concomitant of the $r^{t h}$ GOS. The $p d f$ and $c d f$ of $Y_{[r, n, m, k]}, 1 \leq r \leq n$ the $r^{t h}$ concomitant of GOS, denoted by $g_{[r, n, m, k]}$ and $G_{[r, n, m, k]}$ respectively are given by

$$
g_{[r, n, m, k]}(y)=\int_{-\infty}^{\infty} f_{y \mid x}(y \mid x) f_{r, n, m, k}(x) d x
$$

and

$$
G_{[r, n, m, k]}(y)=\int_{-\infty}^{\infty} F_{y \mid x}(y \mid x) f_{r, n, m, k}(x) d x,
$$

where $f_{r, n, m, k}(x)$ is the $p d f$ of $X(r, n, m, k)$.

Let $Y_{[r, n, m, k]}$ and $Y_{[s, n, m, k]}$ be concomitants of $r^{t h}$ and $s^{t h}$ GOS, respectively. Then the joint $p d f$ and $c d f$ of $Y_{[r, n, m, k]}$ and $Y_{[s, n, m, k]}$ are respectively given by

$$
g_{[r, s n, m, k]}\left(y_{1}, y_{2}\right)=\int_{-\infty}^{\infty} \int_{-\infty}^{x_{2}} f_{y \mid x}\left(y_{1} \mid x_{1}\right) f_{y \mid x}\left(y_{2} \mid x_{2}\right) f_{r, s, n, m, k}\left(x_{1}, x_{2}\right) d x_{1} d x_{2}
$$

and

$$
G_{[r, s n, m, k]}\left(y_{1}, y_{2}\right)=\int_{-\infty}^{\infty} \int_{-\infty}^{x_{2}} F_{y \mid x}\left(y_{1} \mid x_{1}\right) f_{y \mid x}\left(y_{2} \mid x_{2}\right) f_{r, s, n, m, k}\left(x_{1}, x_{2}\right) d x_{1} d x_{2}
$$

where $f_{r, s, n, m, k}\left(x_{1}, x_{2}\right)$ is the joint $p d f$ of $X(r, n, m, k), X(s, n, m, k), 1 \leq r<s \leq n$. 


\section{Concomitants of Generalized Order Statistics}

In this Section, the marginal $p d f$ of concomitants of GOS from bivariate Pareto distribution are considered. For the bivariate Pareto distribution as given in (1.1), in view of (1.3), (1.4), (1.5), (2.2) and (2.4), the marginal $p d f$ of $g_{[r, n, m, k]}$ is given as

$$
\begin{aligned}
g_{[r, n, m, k]}(y) & =\frac{C_{r-1}}{(r-1) !} \int_{0}^{\infty} \frac{(\theta+1) \alpha_{2}\left(1+\alpha_{1} x\right)^{\theta+1}}{\left(1+\alpha_{1} x+\alpha_{2} y\right)^{\theta+2}}[\bar{F}(x)]^{\gamma_{r}-1} f(x) g_{m}^{r-1}(F(x)) d x \\
& =\frac{\theta(\theta+1) \alpha_{2} C_{r-1}}{(r-1) !(m+1)^{r-1}} \sum_{u=0}^{r-1}(-1)^{u}\left(\begin{array}{c}
r-1 \\
u
\end{array}\right) \int_{0}^{\infty} \frac{\alpha_{1}}{\left(1+\alpha_{1} x+\alpha_{2} y\right)^{\theta+2}\left(1+\alpha_{1} x\right)^{\theta\left(\gamma_{r-u}-1\right)}} d x
\end{aligned}
$$

Making the substitution $z=\alpha_{1} x(3.1)$, we get

$$
\begin{aligned}
g_{[r, n, m, k]}(y) & =\frac{\theta(\theta+1) \alpha_{2} C_{r-1}}{(r-1) !(m+1)^{r-1}} \sum_{u=0}^{r-1}(-1)^{u}\left(\begin{array}{c}
r-1 \\
u
\end{array}\right) \int_{0}^{\infty}\left(1+z+\alpha_{2} y\right)^{-(\theta+2)}(1+z)^{-\theta\left(\gamma_{r-u}-1\right)} d z \\
& =\frac{\theta(\theta+1) \alpha_{2} C_{r-1}}{(r-1) !(m+1)^{r-1}} \sum_{u=0}^{r-1}(-1)^{u}\left(\begin{array}{c}
r-1 \\
u
\end{array}\right) \frac{\left(1+\alpha_{2} y\right)^{-(\theta+1)}}{\left(\theta \gamma_{r-u}+1\right)} \\
& \times{ }_{2} F_{1}\left[\begin{array}{cc}
\theta \gamma_{r-u}-\theta, & 1 \\
\theta \gamma_{r-u}+2 & ;-\alpha_{2} y
\end{array}\right],
\end{aligned}
$$

where ${ }_{2} F_{1}\left[\begin{array}{ccc}a, & b & ; z \\ c & \end{array}\right]$ denotes the Gauss hypergeometric function defined by

$$
{ }_{2} F_{1}\left[\begin{array}{ccc}
a, & b & ; z \\
c & & ;
\end{array}\right]=\sum_{p=0}^{\infty} \frac{(a)_{p}(b)_{p}}{(c)_{p}} \frac{z^{p}}{p !}
$$

where $(e)_{k}=e(e+1) \cdots(e+k-1)$ denotes the ascending factorial. In-built routines for computing these special functions are available in packages like Maple, Matlab and Mathematica. For example, Hypergeometric ${ }_{2} F_{1}\left[\begin{array}{ccc}a, & b & ; z \\ c & & ;\end{array}\right]$ in Mathematica computes the Gauss hypergeometric function. Using the definition, (3.3), we can rewrite (3.2)

$$
\begin{aligned}
g_{[r, n, m, k]}(y) & =\frac{\theta(\theta+1) \alpha_{2} C_{r-1}}{(r-1) !(m+1)^{r-1}} \sum_{u=0}^{r-1}(-1)^{u}\left(\begin{array}{c}
r-1 \\
u
\end{array}\right) \frac{1}{\left(\theta \gamma_{r-u}+1\right)} \\
& \times \sum_{p=0}^{\infty} \frac{\left(\theta \gamma_{r-u}-\theta\right)_{p}(1)_{p}}{\left(\theta \gamma_{r-u}+2\right)_{p}} \frac{\left(1+\alpha_{2} y\right)^{-(\theta+1)}\left(-\alpha_{2} y\right)^{p}}{p !} .
\end{aligned}
$$

\section{Single moments of concomitants of generalized order statistics}

Here, we derive explicit expressions for single moments of concomitants of GOS from bivariate Pareto distribution. Using the results of the previous section, we derive the moments of $Y_{[r, n, m, k]}$ as follows:

For the bivariate Pareto distribution given in (1.1), the $j^{\text {th }}$ moment of $Y_{[r, n, m, k]}$ is given as 


$$
\begin{aligned}
E\left(Y_{[r, n, m, k]}^{j}\right) & =\int_{-\infty}^{\infty} y^{j} g_{[r, n, m, k]}(y) d y \\
& =\frac{\theta(\theta+1) \alpha_{2} C_{r-1}}{(r-1) !(m+1)^{r-1}} \sum_{p=0}^{\infty} \sum_{u=0}^{r-1}(-1)^{u}\left(\begin{array}{c}
r-1 \\
u
\end{array}\right) \frac{\left(\theta \gamma_{r-u}-\theta\right)_{p}(1)_{p}}{p !\left(\theta \gamma_{r-u}+1\right)\left(\theta \gamma_{r-u}+2\right)_{p}} \\
& \times \int_{0}^{\infty} y^{j}\left(1+\alpha_{2} y\right)^{-(\theta+1)}\left(-\alpha_{2} y\right)^{p} d y \\
& =\frac{\theta(\theta+1) C_{r-1}}{\alpha_{2}^{j}(r-1) !(m+1)^{r-1}} \sum_{p=0}^{\infty} \sum_{u=0}^{r-1}(-1)^{u+p}\left(\begin{array}{c}
r-1 \\
u
\end{array}\right) \frac{\left(\theta \gamma_{r-u}-\theta\right)_{p}(1)_{p}}{p !\left(\theta \gamma_{r-u}+1\right)\left(\theta \gamma_{r-u}+2\right)_{p}} \\
& \times \int_{1}^{\infty} z^{-(\theta+1)}(z-1)^{j+p} d z,
\end{aligned}
$$

where $z=1+\alpha_{2} y$ and using equation (3.191.2) in Gradshteyn and Ryzhik (2000) the equation (4.1) reduced to.

$$
\begin{aligned}
E\left(Y_{[r, n, m, k]}^{j}\right) & =\frac{\theta(\theta+1) C_{r-1}}{\alpha_{2}^{j}(r-1) !(m+1)^{r-1}} \sum_{p=0}^{\infty} \sum_{u=0}^{r-1}(-1)^{u+p}\left(\begin{array}{c}
r-1 \\
u
\end{array}\right) \\
& \times \frac{\left(\theta \gamma_{r-u}-\theta\right)_{p}(1)_{p} \Gamma(\theta-j-p) \Gamma(j+p+1)}{p !\left(\theta \gamma_{r-u}+1\right)\left(\theta \gamma_{r-u}+2\right)_{p} \Gamma(\theta+1)} \\
& =\frac{\theta(\theta+1) C_{r-1}}{\alpha_{2}^{j}(r-1) !(m+1)^{r-1}} \sum_{p=0}^{\infty} \sum_{u=0}^{r-1}(-1)^{u+p}\left(\begin{array}{c}
r-1 \\
u
\end{array}\right) \\
& \times \frac{\left(\theta \gamma_{r-u}-\theta\right)_{p}(1)_{p}(j+1)_{p} \Gamma(j+1) \Gamma(\theta-j)}{p !\left(\theta \gamma_{r-u}+1\right)\left(\theta \gamma_{r-u}+2\right)_{p}(1-\theta+j)_{p} \Gamma(\theta+1)} .
\end{aligned}
$$

We have generalized Gauss series or the generalized hypergeometric series and see Prudnikov et al., (1986) then the (4.2) reduced to

$$
\begin{aligned}
E\left(Y_{[r, n, m, k]}^{j}\right) & =\frac{C_{r-1}}{\alpha_{2}^{j}(r-1) !(m+1)^{r}} \sum_{u=0}^{r-1}(-1)^{u}\left(\begin{array}{c}
r-1 \\
u
\end{array}\right) \frac{\Gamma(\theta+1-j) \Gamma(j+1)}{\Gamma(\theta+1)\left(\gamma_{r-u}-\frac{j}{\theta}\right)} \\
& =\frac{C_{r-1}}{\alpha_{2}^{j}(r-1) !(m+1)^{r}} \sum_{u=0}^{r-1}(-1)^{u}\left(\begin{array}{c}
r-1 \\
u
\end{array}\right) \frac{\Gamma(\theta+1-j) \Gamma(j+1)}{\Gamma(\theta+1)} \\
& \times B\left(\frac{k}{m+1}+n-r+u-\frac{j}{\theta(m+1)}, 1\right) .
\end{aligned}
$$

since

$$
\sum_{a=0}^{b}(-1)^{a}\left(\begin{array}{l}
b \\
a
\end{array}\right) B(a+k, c)=B(k, c+b),
$$

where $B(a, b)$ is the complete beta function.

Therefore,

$$
\begin{aligned}
E\left(Y_{[r, n, m, k]}^{j}\right) & =\frac{C_{r-1}}{\alpha_{2}^{j}(m+1)^{r}} \frac{\Gamma(\theta+1-j) \Gamma(j+1) \Gamma\left[\frac{k+(n-r)(m+1)-(j / \theta)}{m+1}\right]}{\Gamma(\theta+1) \Gamma\left[\frac{k+n(m+1)-(j / \theta)}{m+1}\right]} \\
& =\frac{\Gamma(\theta+1-j) \Gamma(j+1)}{\alpha_{2}^{j} \Gamma(\theta+1)} \frac{1}{\prod_{i=1}^{r}\left(1-\frac{j}{\theta \gamma_{i}}\right)} .
\end{aligned}
$$

Remark 4.1. Putting $m=0, k=1$ in (4.5), we get moments of order statistics from bivariate Pareto distribution as;

$$
E\left(Y_{[r: n]}^{j}\right)=\frac{n ! \Gamma(\theta+1-j) \Gamma(j+1) \Gamma[n-r+1-(j / \theta)]}{\alpha_{2}^{j}(n-r) ! \Gamma(\theta+1) \Gamma[n+1-(j / \theta)]} .
$$


For $r=n$

$$
E\left(Y_{[n: n]}^{j}\right)=\frac{n ! \Gamma(\theta+1-j) \Gamma(j+1) \Gamma[1-(j / \theta)]}{\alpha_{2}^{j} \Gamma(\theta+1) \Gamma[n+1-(j / \theta)]}
$$

Remark 4.2. Putting $m=-1$ in (4.6), to get moments of $k^{t h}$ record values from bivariate Pareto distribution as;

$$
E\left(Y_{[r, n,-1, k]}^{j}\right)=\frac{\Gamma(\theta+1-j) \Gamma(j+1)}{\alpha_{2}^{j} \Gamma(\theta+1)\left(1-\frac{j}{\theta k}\right)^{r}},
$$

and hence for upper records

$$
E\left(Y_{[U(r)]}^{j}\right)=\frac{\Gamma(\theta+1-j) \Gamma(j+1)}{\alpha_{2}^{j} \Gamma(\theta+1)\left(1-\frac{j}{\theta}\right)^{r}}
$$

\section{Joint distribution of two concomitants of generalized order statistics}

In this Section, the joint $p d f$ of concomitants of GOS from bivariate Pareto distribution are considered. For the bivariate Pareto distribution as given in (1.1), in views of (1.3), (1.4), (1.5), (2.3), and (2.6), the joint $p d f$ of $Y_{[r, n, m, k]}$ and $Y_{[s, n, m, k]}$ is given by

$$
\begin{aligned}
g_{[r, s, n, m, k]}\left(y_{1}, y_{2}\right) & =\frac{\left[\alpha_{2} \theta(\theta+1)^{2}\right] C_{s-1}}{(r-1) !(s-r-1) !} \int_{-\infty}^{\infty} \int_{-\infty}^{x_{2}} \frac{\left(1+\alpha_{1} x_{1}\right)^{\theta+1}\left(1+\alpha_{2} x_{1}\right)^{\theta+1}}{\left(1+\alpha_{1} x_{1}+\alpha_{2} y_{1}\right)^{\theta+2}\left(1+\alpha_{1} x_{2}+\alpha_{2} y_{2}\right)^{\theta+2}} \\
& \times[\bar{F}(x)]^{m} f(x) g_{m}^{r-1}(F(x))\left[h_{m}(F(y))-h_{m}(F(x))\right]^{s-r-1}[\bar{F}(y)]^{\gamma_{s-1}} f(y) \\
& =\frac{\alpha_{1}\left[\alpha_{2} \theta(\theta+1)^{2}\right] C_{s-1}}{(r-1) !(s-r-1) !(m+1)^{s-2}} \sum_{u=0}^{r-1} \sum_{v=0}^{s-r-1}(-1)^{u+v}\left(\begin{array}{c}
r-1 \\
u
\end{array}\right)\left(\begin{array}{c}
s-r-1 \\
u
\end{array}\right) \\
& \times \int_{0}^{\infty} \frac{1}{\left(1+\alpha_{1} x_{1}\right)^{\theta(s-r+u-v)(m+1)-\theta}\left(1+\alpha_{1} x_{1}+\alpha_{2} y_{1}\right)^{\theta+2}} G\left(x_{1}, y_{2}\right) d x_{1},
\end{aligned}
$$

where

$$
G\left(x_{1}, y_{2}\right)=\int_{x_{1}}^{\infty} \frac{\alpha_{1}}{\left(1+\alpha_{1} x_{2}\right)^{\theta\left(\gamma_{s-v}-1\right)}\left(1+\alpha_{1} x_{1}+\alpha_{2} y_{2}\right)^{\theta+2}} d x_{2} .
$$

By setting $t=1+\alpha_{1} x_{2}$ in (5.2), and after simplification we get

$$
G\left(x_{1}, y_{2}\right)=\sum_{p=0}^{\infty}(-1)^{p} \frac{(\theta+2)_{p}\left(1+\alpha_{1} x_{1}\right)^{-\left[\theta\left(\gamma_{s-v}-1\right)-p-1\right]}}{\left(\alpha_{2} y_{2}\right)^{p+\theta+2} p !\left[1-\theta\left(\gamma_{s-v}-1\right)+p\right]} .
$$

On substituting the above expression of $G\left(x_{1}, y_{2}\right)$ in (5.1), and simplifying the resulting equation, we get

$$
\begin{aligned}
g_{[r, s, n, m, k]}\left(y_{1}, y_{2}\right) & =\frac{\left[\theta(\theta+1)^{2}\right] C_{s-1}}{(r-1) !(s-r-1) !(m+1)^{s-2}} \sum_{u=0}^{r-1} \sum_{v=0}^{s-r-1}(-1)^{u+v}\left(\begin{array}{c}
r-1 \\
u
\end{array}\right)\left(\begin{array}{c}
s-r-1 \\
u
\end{array}\right) \\
& \times \frac{1}{\left[1-\theta\left(\gamma_{s-v}-1\right)\right]\left[2-\theta\left(\gamma_{s-v}-2\right)\right]}\left[\frac{\alpha_{2}}{\left(\alpha_{2} y_{2}\right)^{\theta+2}}\right]\left[\frac{\alpha_{2}}{\left(\alpha_{2} y_{1}\right)^{\theta+2}}\right] \\
& \times \sum_{p=0}^{\infty} \sum_{q=0}^{\infty} \frac{\left.\left(2-\theta\left(\gamma_{r-u}-2\right)\right)_{p+q}(\theta+2)\right)_{p}\left(1-\theta\left(\gamma_{s-v}-1\right)\right)_{p}(\theta+2)_{q}}{\left(3-\theta\left(\gamma_{r-u}-2\right)\right)_{p+q}\left(2-\theta\left(\gamma_{r-u}-1\right)\right)_{p}} \\
& \times \frac{1}{p ! q !}\left(-\frac{1}{\alpha_{2} y_{2}}\right)^{p}\left(-\frac{1}{\alpha_{2} y_{1}}\right)^{q} .
\end{aligned}
$$




\section{Product moments of two concomitants of generalized order statistics}

In this Section, the explicit expressions for product moments of two concomitants of GOS from bivariate Pareto distribution are considered. Using the results of the previous section, we derive the product moments of $Y_{[r, n, m, k]}$ and $Y_{[s, n, m, k]}$ as follows:

For the bivariate Pareto distribution given in (1.1), the $i^{t h}$ and $j^{t h}$ moments of $Y_{[r, n, m, k]}$ and $Y_{[s, n, m, k]}$ is given as

$$
\begin{aligned}
E\left(Y_{[r, n, m, k]}^{i}, Y_{[s, n, m, k]}^{j}\right) & =\int_{0}^{\infty} \int_{y_{2}}^{\infty} y_{1}^{i} y_{2}^{j} g_{[r, s, n, m, k]}\left(y_{1}, y_{2}\right) d y_{1} d y_{2} \\
& =A \int_{0}^{\infty} \int_{y_{2}}^{\infty} y_{1}^{i} y_{2}^{j}\left[\frac{\alpha_{2}}{\left(\alpha_{2} y_{2}\right)^{\theta+2}}\right]\left[\frac{\alpha_{2}}{\left(\alpha_{2} y_{1}\right)^{\theta+2}}\right] \sum_{p=0}^{\infty} \sum_{q=0}^{\infty} \frac{\left(2-\theta\left(\gamma_{r-u}-2\right)\right)_{p+q}}{\left(3-\theta\left(\gamma_{r-u}-2\right)\right)_{p+q}} \\
& \times \frac{(\theta+2)_{p}\left(1-\theta\left(\gamma_{s-v}-1\right)\right)_{p}(\theta+2)_{q}}{p ! q !\left(2-\theta\left(\gamma_{r-u}-1\right)\right)_{p}} d y_{1} d y_{2},
\end{aligned}
$$

where

$$
\begin{aligned}
A & =\frac{\left[\theta(\theta+1)^{2}\right] C_{s-1}}{(r-1) !(s-r-1) !(m+1)^{s-2}} \sum_{u=0}^{r-1} \sum_{v=0}^{s-r-1}(-1)^{u+v}\left(\begin{array}{c}
r-1 \\
u
\end{array}\right)\left(\begin{array}{c}
s-r-1 \\
u
\end{array}\right) \\
& \times \frac{1}{\left[1-\theta\left(\gamma_{s-v}-1\right)\right]\left[2-\theta\left(\gamma_{s-v}-2\right)\right]} .
\end{aligned}
$$

By Srivastava and Karlsson (1985) and the setting $z-1=\alpha_{2} y_{1}$ in (6.1), we have after simplification

$$
\begin{aligned}
E\left(Y_{[r, n, m, k]}^{i}, Y_{[s, n, m, k]}^{j}\right) & =\frac{\left[\theta(\theta+1)^{2}\right] C_{s-1} \Gamma(\theta+1-i) \Gamma(i+1) \Gamma(\theta+1-j) \Gamma(j+1)}{\alpha_{2}^{i+j}(r-1) !(s-r-1) !(m+1)^{s-2} \Gamma(\theta+2) \Gamma(\theta+2)} \\
& \times \sum_{u=0}^{r-1} \sum_{v=0}^{s-r-1}\left(\begin{array}{c}
r-1 \\
u
\end{array}\right)\left(\begin{array}{c}
s-r-1 \\
u
\end{array}\right) \frac{(-1)^{u+v}}{[\theta(k+(n-s+v)(m+1))-j]} \\
& \times \frac{1}{[\theta(k+(n-r+u)(m+1))-(i+j)]} \\
& =\frac{\left[\theta(\theta+1)^{2}\right] C_{s-1} \Gamma(\theta+1-i) \Gamma(i+1) \Gamma(\theta+1-j) \Gamma(j+1)}{\alpha_{2}^{i+j}(r-1) !(s-r-1) !(m+1)^{s} \Gamma(\theta+2) \Gamma(\theta+2)} \\
& \times \sum_{u=0}^{r-1}(-1)^{u}\left(\begin{array}{c}
r-1 \\
u
\end{array}\right) B\left(\frac{k}{m+1}+n-r+u-\frac{i+j}{\theta(m+1)}, 1\right) \\
& \times \sum_{v=0}^{s-r-1}(-1)^{v}\left(\begin{array}{c}
s-r-1 \\
v
\end{array}\right) B\left(\frac{k}{m+1}+n-s+v-\frac{j}{\theta(m+1)}, 1\right) .
\end{aligned}
$$

On using relation (4.4) in (6.2), and simplifying the resulting expression we get

$$
\begin{aligned}
E\left(Y_{[r, n, m, k]}^{i}, Y_{[s, n, m, k]}^{j}\right) & =\frac{\left[\theta(\theta+1)^{2}\right] C_{s-1} \Gamma(\theta+1-i) \Gamma(i+1) \Gamma(\theta+1-j) \Gamma(j+1)}{\alpha_{2}^{i+j}(r-1) !(s-r-1) !(m+1)^{s} \Gamma(\theta+2) \Gamma(\theta+2)} \\
& \times B\left(\frac{k}{m+1}+n-r-\frac{i+j}{\theta(m+1)}, r\right) B\left(\frac{k}{m+1}+n-s-\frac{j}{\theta(m+1)}, s-r\right) \\
& =\frac{\Gamma(\theta+1-i) \Gamma(i+1) \Gamma(\theta+1-j) \Gamma(j+1)}{\alpha_{2}^{i+j} \Gamma(\theta+2) \Gamma(\theta+2)} \\
& \times \frac{1}{\prod_{a=1}^{r}\left(1+\frac{i+j}{\theta \gamma_{a}}\right) \prod_{b=r+1}^{s}\left(1+\frac{j}{\theta \gamma_{b}}\right)}
\end{aligned}
$$

Remark 6.1. Putting $m=0, k=1$ in (6.3), we get product moments of order statistics from bivariate Pareto 
distribution as;

$$
\begin{aligned}
E\left(Y_{[r: n]}^{i}, Y_{[s: n]}^{j}\right) & =\frac{n ! \Gamma(\theta+1-i) \Gamma(i+1) \Gamma(\theta+1-j) \Gamma(j+1)}{(n-s) ! \alpha_{2}^{i+j} \Gamma(\theta+2) \Gamma(\theta+2)} \\
& \times \frac{\Gamma\left(n-r+1-\frac{i+j}{\theta}\right) \Gamma\left(n-s+1-\frac{j}{\theta}\right)}{\Gamma\left(n+1-\frac{i+j}{\theta}\right) \Gamma\left(n-r+1-\frac{j}{\theta}\right)} .
\end{aligned}
$$

Remark 6.2. Putting $m=-1$ in (6.4), we get the product moments of $k^{t h}$ record values from bivariate Pareto distribution as;

$$
E\left(Y_{[r, n,-1, k]}^{i}, Y_{[s, n,-1, k]}^{j}\right)=\frac{\Gamma(\theta+1-i) \Gamma(i+1) \Gamma(\theta+1-j) \Gamma(j+1)}{\alpha_{2}^{i+j} \Gamma(\theta+2) \Gamma(\theta+2)} \frac{1}{\left(1+\frac{i+j}{\theta k}\right)^{r}\left(1+\frac{j}{\theta k}\right)^{s-r}}
$$

and hence for upper records

$$
E\left(Y_{[r, n,-1, k]}^{i}, Y_{[s, n,-1, k]}^{j}\right)=\frac{\Gamma(\theta+1-i) \Gamma(i+1) \Gamma(\theta+1-j) \Gamma(j+1)}{\alpha_{2}^{i+j} \Gamma(\theta+2) \Gamma(\theta+2)} \frac{1}{\left(1+\frac{i+j}{\theta}\right)^{r}\left(1+\frac{j}{\theta}\right)^{s-r}} .
$$

\section{Numerical results and discussion}

The explicit expressions obtained in the preceding sections allow us to evaluate the means, variances and covariances of all concomitants of order statistics for all sample sizes. The mean and variances of concomitant order statistics of a bivariate Pareto distribution for different values of $\theta$ and $\alpha_{2}$ are calculated in Tables 1-2, respectively. The mean and variances of concomitant of record values of bivariate Pareto distribution for different values of $\theta$ and $\alpha_{2}$ are calculated in Tables 3-4, respectively. It appears from the results that the mean of concomitant of order statistics and record values decrease with both $\theta$ and $\alpha_{2}$. Means, variances and covariances of all order statistics can be used for various inferential purposes; for example, they are useful in determining best linear unbiased estimators

\begin{tabular}{|c|c|c|c|c|c|c|c|c|c|c|}
\hline \multirow[b]{2}{*}{$\mathrm{n}$} & \multirow{2}{*}{$\mathrm{r}$} & \multicolumn{3}{|c|}{$\theta=2$} & \multicolumn{3}{|c|}{$\theta=3$} & \multicolumn{3}{|c|}{$\theta=4$} \\
\hline & & $\alpha_{2}=1$ & $\alpha_{2}=2$ & $\alpha_{2}=3$ & $\alpha_{2}=1$ & $\alpha_{2}=2$ & $\alpha_{2}=3$ & $\alpha_{2}=1$ & $\alpha_{2}=2$ & $\alpha_{2}=3$ \\
\hline 1 & 1 & 0.500000 & 0.250000 & 0.166667 & 0.333333 & 0.166667 & 0.111111 & 0.250000 & 0.125000 & 0.083333 \\
\hline \multirow[b]{2}{*}{2} & 1 & 0.400000 & 0.200000 & 0.133333 & 0.285714 & 0.142857 & 0.095238 & 0.222222 & 0.111111 & 0.074074 \\
\hline & 2 & 0.600000 & 0.300000 & 0.200000 & 0.380952 & 0.190476 & 0.126984 & 0.277778 & 0.138889 & 0.092593 \\
\hline \multirow{3}{*}{3} & 1 & 0.375000 & 0.187500 & 0.125000 & 0.272727 & 0.136364 & 0.090909 & 0.214286 & 0.107143 & 0.071429 \\
\hline & 2 & 0.450000 & 0.225000 & 0.150000 & 0.311688 & 0.155844 & 0.103896 & 0.238095 & 0.119048 & 0.079365 \\
\hline & 3 & 0.675000 & 0.337500 & 0.225000 & 0.415584 & 0.207792 & 0.138528 & 0.297619 & 0.148810 & 0.099206 \\
\hline \multirow{4}{*}{4} & 1 & 0.363636 & 0.181818 & 0.121212 & 0.266667 & 0.133333 & 0.088889 & 0.210526 & 0.105263 & 0.070175 \\
\hline & 2 & 0.409091 & 0.204545 & 0.136364 & 0.290909 & 0.145455 & 0.096970 & 0.225564 & 0.112782 & 0.075188 \\
\hline & 3 & 0.490909 & 0.245455 & 0.163636 & 0.332468 & 0.166234 & 0.110823 & 0.250627 & 0.125313 & 0.083542 \\
\hline & 4 & 0.736364 & 0.368182 & 0.245455 & 0.443290 & 0.221645 & 0.147763 & 0.313283 & 0.156642 & 0.104428 \\
\hline \multirow{5}{*}{5} & 1 & 0.357143 & 0.178571 & 0.119048 & 0.263158 & 0.131579 & 0.087719 & 0.208333 & 0.104167 & 0.069444 \\
\hline & 2 & 0.389610 & 0.194805 & 0.129870 & 0.280702 & 0.140351 & 0.093567 & 0.219298 & 0.109649 & 0.073099 \\
\hline & 3 & 0.438312 & 0.219156 & 0.146104 & 0.306220 & 0.153110 & 0.102073 & 0.234962 & 0.117481 & 0.078321 \\
\hline & 4 & 0.525974 & 0.262987 & 0.175325 & 0.349966 & 0.174983 & 0.116655 & 0.261069 & 0.130535 & 0.087023 \\
\hline & 5 & 0.788961 & 0.394481 & 0.262987 & 0.466621 & 0.233311 & 0.155540 & 0.326337 & 0.163168 & 0.108779 \\
\hline
\end{tabular}
of location/scale parameters and best linear unbiased predictors of failure times. More details on BLUEs and BLUPs based on order statistics can be seen in Balakrishnan and Cohen (1991) and Arnold et al. (1992).

Table 1. Means of the concomitant of order statistics 
Table 2. Variances of the concomitant of order statistics

\begin{tabular}{|c|c|c|c|c|c|c|c|c|c|c|}
\hline & \multirow{2}{*}{$r$} & \multicolumn{3}{|c|}{$\theta=2$} & \multicolumn{3}{|c|}{$\theta=3$} & \multicolumn{3}{|c|}{$\theta=4$} \\
\hline $\mathrm{n}$ & & $\alpha_{2}=1$ & $\alpha_{2}=2$ & $\alpha_{2}=3$ & $\alpha_{2}=1$ & $\alpha_{2}=2$ & $\alpha_{2}=3$ & $\alpha_{2}=1$ & $\alpha_{2}=2$ & $\alpha_{2}=3$ \\
\hline 1 & 1 & 0.750000 & 0.187500 & 0.083333 & 0.222222 & 0.055555 & 0.024691 & 0.104167 & 0.026042 & 0.011575 \\
\hline \multirow{2}{*}{2} & 1 & 0.340000 & 0.085000 & 0.037778 & 0.140590 & 0.035148 & 0.015621 & 0.075617 & 0.018904 & 0.008402 \\
\hline & 2 & 1.140000 & 0.285000 & 0.126667 & 0.299320 & 0.074830 & 0.033258 & 0.131172 & 0.032793 & 0.014575 \\
\hline \multirow{3}{*}{3} & 1 & 0.287946 & 0.071987 & 0.031994 & 0.125620 & 0.031405 & 0.013958 & 0.069467 & 0.017366 & 0.007719 \\
\hline & 2 & 0.440357 & 0.110089 & 0.0 & 0.169518 & 0.04238 & 836 & 0.087542 & 0.021886 & 0.009727 \\
\hline & 3 & 1.472946 & 0.368237 & 0.163661 & 0.360623 & 0.090155 & 0.040069 & 0.151808 & 0.037952 & 0.016867 \\
\hline \multirow{4}{*}{4} & 1 & 0.267769 & 0.066942 & 0.029752 & 0.119365 & 0.029841 & 0.013263 & 0.066791 & 0.016698 & 0.007421 \\
\hline & 2 & 0.346931 & 0.086732 & 0.038548 & 0.143943 & 0.035986 & 0.015994 & 0.077326 & 0.019331 & 0.008592 \\
\hline & 3 & 0.530437 & 0.132609 & 0.058937 & 0.194227 & 0.048556 & 0.021581 & 0.097442 & 0.024361 & 0.010827 \\
\hline & 4 & 1.772054 & 0.443013 & 0.196895 & 0.413018 & 0.103254 & 0.045891 & 0.168948 & 0.042237 & 0.018772 \\
\hline \multirow{5}{*}{5} & 1 & 0.257064 & 0.064266 & 0.028563 & 0.115933 & 0.028983 & 0.012881 & 0.065293 & 0.016323 & 0.007255 \\
\hline & 2 & 0.309742 & 0.077436 & 0.034416 & 0.132846 & 0.033212 & 0.014761 & 0.072681 & 0.018170 & 0.008076 \\
\hline & 3 & 0.401290 & 0.100323 & 0.0 & 0.16 & 0.0 & 310 & 0.084146 & 0.021036 & 0.009350 \\
\hline & 4 & 0.613461 & 0.153365 & 0.0 & 0.21 & 0.05 & 017 & 0.106035 & 0.026509 & 0.011782 \\
\hline & 5 & 2.047871 & 0.511967 & 0.227541 & 0.459514 & 0.114878 & 0.051057 & 0.183824 & 0.045956 & 0.020425 \\
\hline
\end{tabular}

Table 3. Means of the concomitant of record values

\begin{tabular}{|c|l|l|l|l|l|l|l|l|l|}
\hline \multirow{2}{*}{$\mathrm{r}$} & \multicolumn{3}{|c|}{$\theta=2$} & \multicolumn{3}{c|}{$\theta=3$} & \multicolumn{3}{c|}{$\theta=4$} \\
\cline { 2 - 10 } & $\alpha_{2}=1$ & $\alpha_{2}=2$ & $\alpha_{2}=3$ & $\alpha_{2}=1$ & $\alpha_{2}=2$ & $\alpha_{2}=3$ & $\alpha_{2}=1$ & $\alpha_{2}=2$ & $\alpha_{2}=3$ \\
\hline 1 & 0.500000 & 0.250000 & 0.166667 & 0.333333 & 0.166667 & 0.111111 & 0.250000 & 0.125000 & 0.083333 \\
\hline 2 & 0.750000 & 0.375000 & 0.250000 & 0.444444 & 0.222222 & 0.148148 & 0.312500 & 0.156250 & 0.104167 \\
\hline 3 & 1.125000 & 0.562500 & 0.375000 & 0.592593 & 0.296296 & 0.197531 & 0.390625 & 0.195312 & 0.130208 \\
\hline 4 & 1.687500 & 0.843750 & 0.562500 & 0.790123 & 0.395062 & 0.263374 & 0.488281 & 0.244141 & 0.162760 \\
\hline 5 & 2.531250 & 1.265625 & 0.843750 & 1.053498 & 0.526749 & 0.351166 & 0.610352 & 0.305176 & 0.203451 \\
\hline 6 & 3.796875 & 1.898437 & 1.265625 & 1.404664 & 0.702332 & 0.468221 & 0.762939 & 0.381470 & 0.254313 \\
\hline 7 & 5.695312 & 2.847656 & 1.898437 & 1.872885 & 0.936443 & 0.624295 & 0.953674 & 0.476837 & 0.317891 \\
\hline 8 & 8.542969 & 4.271484 & 2.847656 & 2.497180 & 1.248590 & 0.832393 & 1.192093 & 0.596046 & 0.397364 \\
\hline 9 & 12.81445 & 6.407227 & 4.271484 & 3.329574 & 1.664787 & 1.109858 & 1.490116 & 0.745058 & 0.496705 \\
\hline 10 & 19.22168 & 9.610840 & 6.407227 & 4.439432 & 2.219716 & 1.479811 & 1.862645 & 0.931323 & 0.620882 \\
\hline
\end{tabular}

Table 4. Variances of the concomitant of record values

\begin{tabular}{|c|l|l|l|l|l|l|l|l|l|}
\hline \multirow{2}{*}{$\mathrm{r}$} & \multicolumn{3}{|c|}{$\theta=2$} & \multicolumn{3}{c|}{$\theta=3=4$} \\
\cline { 2 - 11 } & $\alpha_{2}=1$ & $\alpha_{2}=2$ & $\alpha_{2}=3$ & $\alpha_{2}=1$ & $\alpha_{2}=2$ & $\alpha_{2}=3$ & $\alpha_{2}=1$ & $\alpha_{2}=2$ & $\alpha_{2}=3$ \\
\hline 1 & 0.750000 & 0.187500 & 0.083333 & 0.222222 & 0.055555 & 0.024691 & 0.104167 & 0.026042 & 0.011575 \\
\hline 2 & 2.437500 & 0.609375 & 0.270833 & 0.469137 & 0.117284 & 0.052126 & 0.180122 & 0.045030 & 0.020013 \\
\hline 3 & 7.734375 & 1.933594 & 0.859375 & 0.982167 & 0.245542 & 0.10913 & 0.310375 & 0.077594 & 0.034486 \\
\hline 4 & 24.15234 & 6.038086 & 2.683594 & 2.042373 & 0.510593 & 0.22693 & 0.533187 & 0.133296 & 0.059243 \\
\hline 5 & 74.59277 & 18.64819 & 8.288086 & 4.223475 & 1.055868 & 0.469275 & 0.913478 & 0.228370 & 0.101498 \\
\hline 6 & 228.5837 & 57.14594 & 25.39819 & 8.693586 & 2.173397 & 0.965954 & 1.561271 & 0.390318 & 0.173475 \\
\hline 7 & 696.5634 & 174.1409 & 77.39594 & 17.82563 & 4.456408 & 1.980626 & 2.662751 & 0.665687 & 0.295861 \\
\hline 8 & 2114.018 & 528.5044 & 234.8909 & 36.43076 & 9.10769 & 4.047863 & 4.532656 & 1.133164 & 0.503629 \\
\hline 9 & 6396.790 & 1599.197 & 710.7544 & 74.24727 & 18.56182 & 8.249696 & 7.702457 & 1.925615 & 0.855829 \\
\hline 10 & 19313.53 & 4828.382 & 2145.947 & 150.9581 & 37.73953 & 16.77312 & 13.06873 & 3.267180 & 1.452081 \\
\hline
\end{tabular}




\section{Applications}

In this Section we suggest some applications

1. Concomitants of order statistics have found a wide variety of applications in different fields. The most important use of concomitants arises in selection procedures when $k(<n)$ individuals are chosen on the basis of their $X$-values. Then the corresponding $Y$ - values represent performance on an associated characteristic. For example, $X$ might be the score of a candidate on a screening test and $Y$ the score on a later test.

1. The single and product moments are given in Section 4 and 6 can be used to calculate the variance covariance matrix.

\section{Conclusions}

In the study presented above, some new explicit expressions for the single and product moments of concomitants of GOS from the Bivariate Pareto distribution has been derived. In addition the means and variances of concomitants of order statistics and records values are calculated for some choices of the parameters. It will be interesting to study the BLUE and BLUP of Bivariate Pareto distribution and to analyze the data. The work is in progress and it will be reported later.

\section{Acknowledgments}

The author would like to thank the reviewers and the editors for their comments which helped improve the paper.

\section{References}

[1] Ahmad, A.A. and Fawzy, M., Recurrence relations for single moments of generalized order statistics from doubly truncated distribution. J. Statist. Plann. Inference, 117 (2003), 241-249.

[2] Ahmad, A.A., Relations for single and product moments of generalized order statistics from doubly truncated Burr type XII distribution. J. Egypt. Math. Soc., 15 (2007), 117-128.

[3] Ahsanullah, M., Generalized order statistics from exponential distribution. J. Statist. Plann. Inference, 85 (2000), 85-91.

[4] Arnold, B. C., Pareto Distribution. Wiley, New York, (1985).

[5] Arnold, B. C., A flexible family of Multivariate Pareto distributions. Journal of Statistical Planning and Inference, 24 (1990), 249-258.

[6] Arnold, B. C., Balakrishnan, N. and Nagaraja, H. N., A First Course in Order Statistics. John Wiley, New York, (1992).

[7] Balakrishnan, N. and Cohen, A. C., Order Statistics and Inference: Estimation Methods. Academic Press, Boston, MA. (1991).

[8] Balakrishnan, N. and Lai, C.D., Continuous bivariate distributions, 2nd-edition, Springer, New York, (2009).

[9] Balakrishnan, N., Zhu, X. and Al-Zahrani, B., A recursive algorithm for the single and product moments of order statistics from the exponential-geometric distribution and some estimation methods. Comm. Statist. Theory Methods, 44 (2015), 3576-3598.

[10] Bieniek, M. and Szynal, D., Characterizations of distributions via linearity of regression of generalized order statistics. Metrika, 58 (2003), 259-271.

[11] Cramer, E., Kamps, U. and Keseling, C., Characterization via linear regression of ordered random variables: a unifying approach. Comm. Statist. Theory Methods, 33 (2004), 2885-2911.

[12] David, H. A. and Nagaraja, H. N., Order Statistics, third edition. John Wiley, New York, (2003). 
[13] Gradshteyn, I. S. and Ryzhik, I. M., Table of Integrals, Series, and Products, sixth edition. Academic Press, San Diego, (2000).

[14] Hutchinson, T. P. and Lai, C. D., Continuous Bivariate Distributions, Emphasising Applications. Rumsby Scientific Publishing, Adelaide, (1990).

[15] Kamps, U., A Concept of Generalized Order Statistics, B.G. Teubner Stuttgart, (1995).

[16] Kamps, U. and Cramer, E., On distributions of generalized order statistics. Statistics, 35 (2001), 269-280.

[17] Kamps, U. and Gather, U., Characteristic property of generalized order statistics for exponential distribution. Appl. Math. (Warsaw), 24 (1997), 383-391.

[18] Keseling, C., Conditional distributions of generalized order statistics and some characterizations. Metrika, 49 (1999), 27-40.

[19] Khan, R. U. and Kumar, D., On moments of lower generalized order statistics from exponentiated Pareto distribution and its characterization, Appl. Math. Sci., 4 (2010), 2711-2722.

[20] Kumar, D., Recurrence relations for single and product moments of generalized order statistics from $p^{\text {th }}$ order exponential distribution and its characterization. Journal of Statistical Research of Iran, 7 (2010), 101-112.

[21] Kumar, D., Generalized order statistics from Kumaraswamy distribution and its characterization. Tamsui Oxford journal of Mathematical Sciences, 27 (2011), 463-476.

[22] Kumar, D., On moments of lower generalized order statistics from exponentiated lomax distribution and characterization. American Journal of Mathematical and Management Sciences, 32 (2013), 238-256.

[23] Kumar, D., Exact moments of generalized order statistics from type II exponentiated log-logistic distribution. Hacettepe Journal of Mathematics and Statistics, 44 (2015a), 715-733.

[24] Kumar, D., Lower generalized order statistics based on inverse Burr distribution, American Journal of Mathematical and Management Sciences, 35(2015b), 15-35.

[25] Langseth, H., Bayesian networks with applications in reliability analysis, Ph.D. thesis, Norwegian University of Science and Technology, Norway, (2002).

[26] Lindley, D.V. and Singpurwalla, N.D., Multivariate distribution for the life lengths of a system sharing a common environment. Journal of Applied Probability, 23 (1986), 418-431.

[27] Mardia, K. V., Multivariate Pareto distributions. The Annals of Mathematical Statistics, 33 (1962), 1008-1015.

[28] Nayak, T., Multivariate Lomax distribution, properties and usefulness in reliability theory. Journal of Applied Probability, 24 (1987), 170-177.

[29] Pawlas, P. and Szynal, D., Recurrence relations for single and product moments of generalized order statistics from Pareto, generalized Pareto, and Burr distributions. Comm. Statist. Theory Methods, 30 (2001), 739-746.

[30] Prudnikov, A.P., Brychkov, Y.A., Marichev, I., Integral and series Vol. 3, More Special Functions, Gordon and Breach Science Publisher, New York, (1986).

[31] Sankaran, P.G. and Nair, N.U., A bivariate Pareto model and its applications to reliability, Naval Research Logistics, 40 (1993), 1013-1020.

[32] Sankaran, P. G. and Kundu, D., A bivariate Pareto model, Statistics, 48 (2014), 241-255.

[33] Sankaran, P. G., Nair, N.U. and John, P., Characterizations of a family of bivariate Pareto distributions, STATISTICA, LXXV (2015), 275-290.

[34] Srivastava, H.M., Karlsson, P.W., Multiple Gaussian Hypergeometric Series, John Wiley and Sons, New York, (1985). 


\section{Affiliations}

\section{DEVENDRA Kumar}

ADDRESS: Department of Statistics

Central University of Haryana

Mahendergarh-123029, India.

E-MAIL: devendrastats@gmail.com 